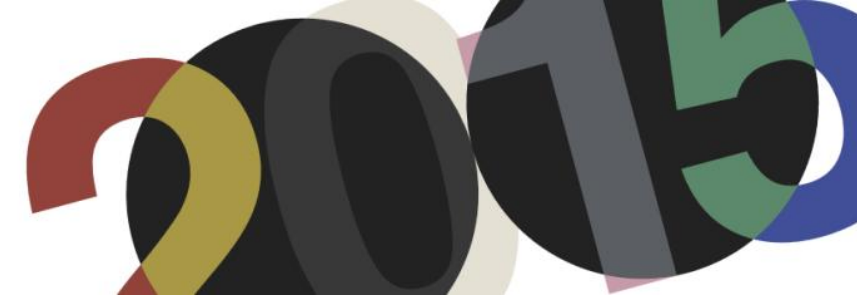

DOI: http://dx.doi.org/10.4995/LC2015.2015.690

\title{
Le Corbusier y la construcción vertical del espacio estratificado
}

\author{
S. Delgado Berrocal
}

\author{
Escuela Superior de Arquitectura de Madrid
}

\begin{abstract}
Resumen: La construcción del espacio mediante la superposición de planos verticales paralelos y franjas espaciales fue empleada por Le Corbusier tanto en sus obras pictóricas como arquitectónicas, tal y como manifestaron Colin Rowe y Robert Slutzky, en 1955-1956, al descomponer y analizar la "transparencia fenoménica" de la Villa Stein en Garches, 1926, y del cuadro Nature morte à la pile d'assiettes, 1920, de Le Corbusier. Un sistema de estratificación espacial vertical mediante superficies opacas, derivado del cubismo, que genera una profundidad bidimensional donde la plástica expresa la plenitud de un volumen sin recurrir a la perspectiva. Una investigación sobre como Le Corbusier emplea la superposición de estratos verticales o de elementos autónomos y espaciados, es decir, de fragmentos de experiencias discontinuas en posiciones relativas, pero fuertemente vinculados entre sí para asegurar la coherencia estable de los resultados prefigurados, pre-determinados, pre-estabilizados. Un recurso donde el uso de relaciones formales directas, y el posicionamiento del objeto, es más importante que la representación del propio objeto. En base a lo cual, la presente comunicación pretende indagar sobre como ocasionar múltiples lecturas de esa nueva espacialidad plana -ampliando los puntos de vista multifocales y las partes independientes, pero manteniendo la unidad compositiva-; y sobre el paso de procesos de transmisión directa de relaciones formales a transformaciones ilegibles que provoquen nuevas emociones.
\end{abstract}

Abstract: The construction of space by overlapping parallel vertical planes and space bands was used by Le Corbusier both in his paintings and architectural projects, just as stated Colin Rowe and Robert Slutzky, in 1955-1956, to break down and analyze the "transparency phenomenal" to the Villa Stein in Garches, 1926, and the painting Nature morte à la pile d'assiettes, 1920, by Le Corbusier. A vertical spatial layering system by opaque surfaces, derivative of cubism, which generates a two-dimensional depth, where the composition expresses the fullness of a volume without resorting to perspective. An investigation about how Le Corbusier employs overlapping vertical layers or autonomous and spaced elements, that is to say, fragments of discontinuous experiences in relative positions, but strongly linked together to ensure stable consistency of the results pre-figured, pre-certain, pre-stabilized. A resource where the use of direct formal relations, and the positioning of the object, is more important than the representation of the object itself. Based on which, the present communication aims to investigate about how to cause multiple readings from this new flat spatiality-increasing multifocal viewpoints and independent parts, while maintaining the compositional unit-; and about the passage from direct transmission processes of formal relations to illegible transformations that cause new emotions.

Palabras clave: Espacio; Estratificación; Transparencia fenoménica; Profundidad bidimensional; Le Corbusier. Keywords: Space; Stratification; Transparency phenomenal; Two-dimensional depth; Le Corbusier.

\section{Superposición de estratos verticales. El espacio en profundidad bidimensional.}

Durante la segunda mitad del s. XX, los arquitectos Colin Rowe y Robert Slutzky, en su escrito Transparency: Literal and Phenomenal ${ }^{l}$, emplearon el concepto de "estratificación" para nombrar un recurso formal de

\footnotetext{
${ }^{1}$ Véase Rowe, Colin y Slutzky, Robert: “Transparency: Literal and Phenomenal.” Escrito en 1955-1956 en colaboración con Robert Slutzky y publicado por primera vez en Perspecta n.8, Yale Architectural Journal, 1963, pp.45-54. Reeditado en Rowe, Colin: Manierismo y arquitectura moderna y otros ensayos, Barcelona: Gustavo Gili, 1978.
} 
"construcción del espacio", basado en los estudios ópticos y las asociaciones provenientes del cubismo, que lograba alcanzar una transparencia fenoménica, es decir, tal y como manifestaban los citados autores, el uso de:

"Estratificaciones: recursos por medio de los cuales el espacio queda construido, substancial, y articulado, son la esencia de aquella transparencia fenomenal [léase fenoménica] que hemos observado como característica central de la tradición poscubista.",2

Un recurso formal relacionado con una nueva concepción del espacio profundo sin recurrir a la perspectiva. Un mecanismo de creación de la profundidad desde un sistema bidimensional, donde, al igual que en los cuadros cubistas, los objetos opacos y aplanados -situados en diversos planos paralelos que los contienen- se superponen y articulan, generando un efecto de transparencia no literal (denominada por Rowe y Slutzky: transparencia fenoménica). Una transparencia no fundamentada en el material del objeto o su condición cristalina -lo que se ve a través-, sino en la lectura o proceso mental de relaciones formales entre objetos opacos -lo que se lee-.

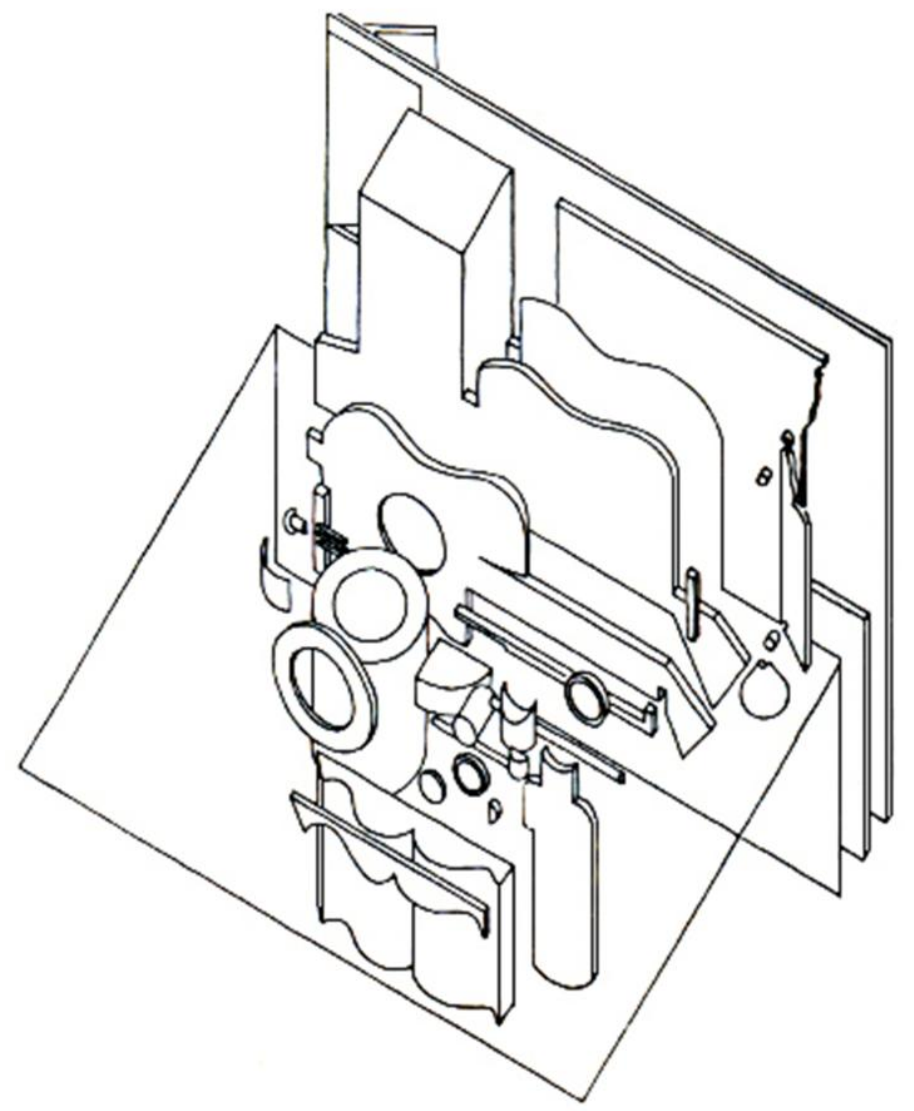

1. Colin Rowe y Robert Slutzky: Descomposición, en una serie de planos paralelos, del cuadro Nature morte à la pile d'assiettes, 1920, de Le Corbusier.

${ }^{2}$ Op. cit.: Rowe, C. y Slutzky, R.: Perspecta, p.53. Texto original: "These stratifications, devices by means of which space becomes constructed, substantial, and articulate, are the essence of that phenomenal transparency witch has been noticed as characteristic of the central postcubist tradition." 

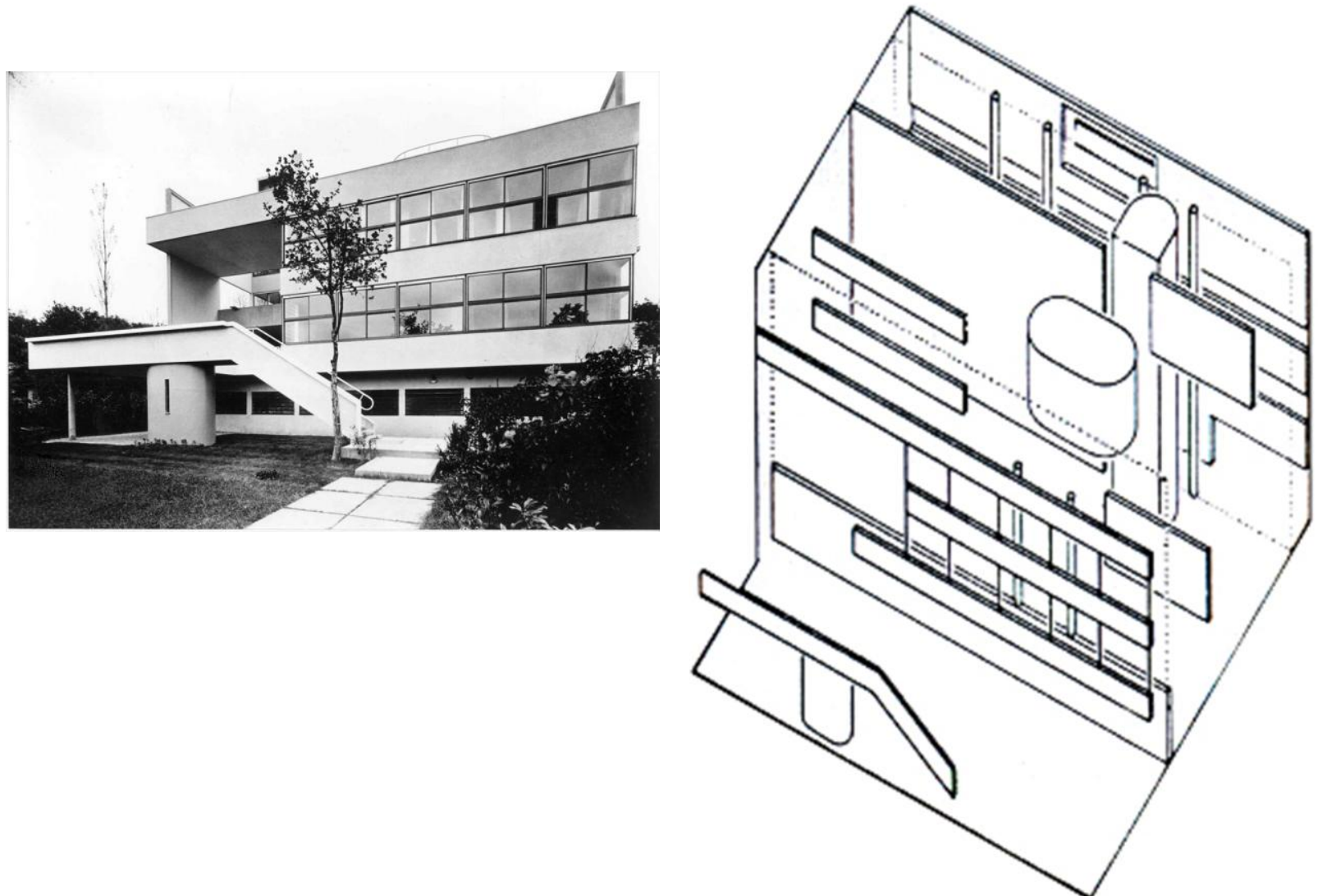

2. Le Corbusier: Villa Stein-de-Monzie, "Les Terrasses”, Garches (Vaucresson), France, 1926. @ FLC/ADAGP

3. Colin Rowe y Robert Slutzky: Descomposición vertical, en una serie de planos paralelos, de la Villa Garches, 1926, de Le Corbusier.

Por consiguiente, se trata de un conjunto de planos paralelos colocados en posiciones relativas siguiendo una estructura lineal, ordenada y legible que organiza una composición unificada, como se puede apreciar en el análisis llevado a cabo por Rowe y Slutzky del cuadro Nature morte à la pile d'assiettes, 1920, (fig.1), y de la Villa Stein en Garches, 1926, (fig.2 y 3), de Le Corbusier.

Unas descomposiciones sobre las que Rowe y Slutzky señalarán:

"Cada uno de estos planos es incompleto en sí mismo o quizás fragmentario; sin embargo la fachada se organiza tomando estos planos paralelos como puntos de referencia, y la implicación total es una estratificación vertical en capas del espacio interior del edificio, una sucesión de espacios que se extienden lateralmente recorriendo uno tras otro. Este sistema de estratificación espacial es lo que relaciona la fachada de Le Corbusier con el cuadro de Léger. "3

\footnotetext{
${ }^{3}$ Op. cit.: Rowe, C. y Slutzky, R.: Perspecta, p.50. Texto original: "Each of these planes is incomplete in itself or perhaps even fragmentary; yet it is with these parallel planes as points of reference that the façade is organized, and the implication of all is of a vertical, layerlike stratification of the interior space of the building, a succession of laterally extended spaces traveling one behind the other. This system of spatial stratification brings Le Corbusier's façade into the closest relationship with the Léger."
} 
Le Corbusier, al igual que Léger en sus cuadros, emplea un método de lectura equívoca de la tridimensionalidad, conseguido mediante un sistema de estratificación espacial.

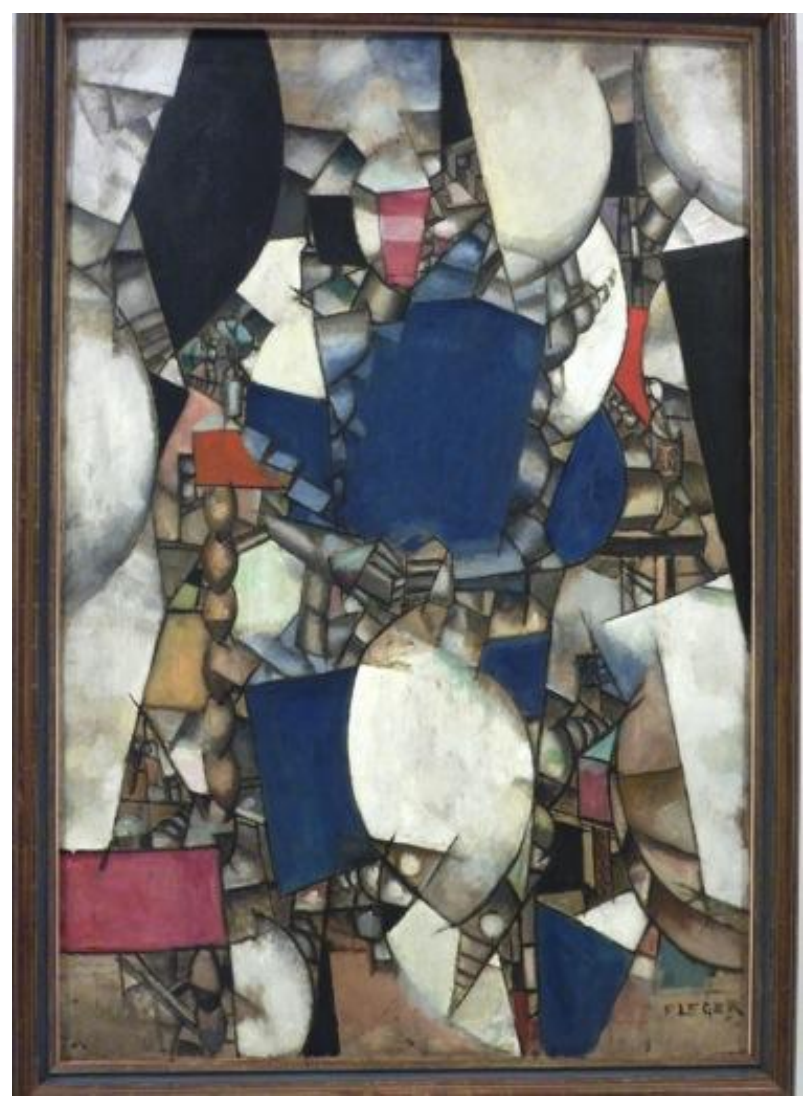

4. Fernand Léger: La mujer de azul, 1912.

En este sentido, Léger intensifica la tensión entre figura y espacio a través de las superposiciones, logrando que la mirada experimente una inacabable serie de organizaciones dentro del todo (fig.4). Para ello articula diversos objetos en un espacio poco profundo y abstracto. Unas superposiciones que proporcionan otra dimensión espacial y llevan a continuas fluctuaciones de la interpretación al no poder abarcarse todos los objetos en una sola mirada.

Asimismo, en los cuadros de Le Corbusier (fig.5), el espacio poco profundo, llano y extendido va adquiriendo poco a poco profundidad, gracias a las superficies paralelas superpuestas que se interpenetran sin la destrucción óptica de los objetos. Tal y como cita George Kepes:

"La representación de la superposición indica profundidad. Crea un sentido al espacio. Cada figura aparece paralelamente al plano gráfico y tiende a establecer una relación espacial de retroceso. "*

Del mismo modo, en la obra arquitectónica de Le Corbusier, se busca la ausencia de volumen, pero no de profundidad -una contradicción entre lo bi- y tri-dimensional-. Se provoca una sensación volumétrica de la fachada -superficie plana-, sugerida mediante la sucesión estratificada de planos abstractos, paralelos, y superpuestos, que dividen verticalmente el espacio (aumentando la extensión interior y persiguiendo la

\footnotetext{
${ }^{4}$ Kepes, Gyorgy: El lenguaje de la visión. Buenos Aires: Infinito, 1969, p.112. (Language of vision. Chicago: Paul Theobald, 1944).
} 
continuidad espacial). La creación de una "función estructural dinámica" con la que se consigue oscilar entre planos autónomos - colocados secuencialmente de forma lineal-, o lo que es lo mismo, donde cada plano actúa como una dirección dinámica independiente que lleva a otro plano, que a su vez lleva a otro, y así consecutivamente hasta la compresión íntegra del conjunto.

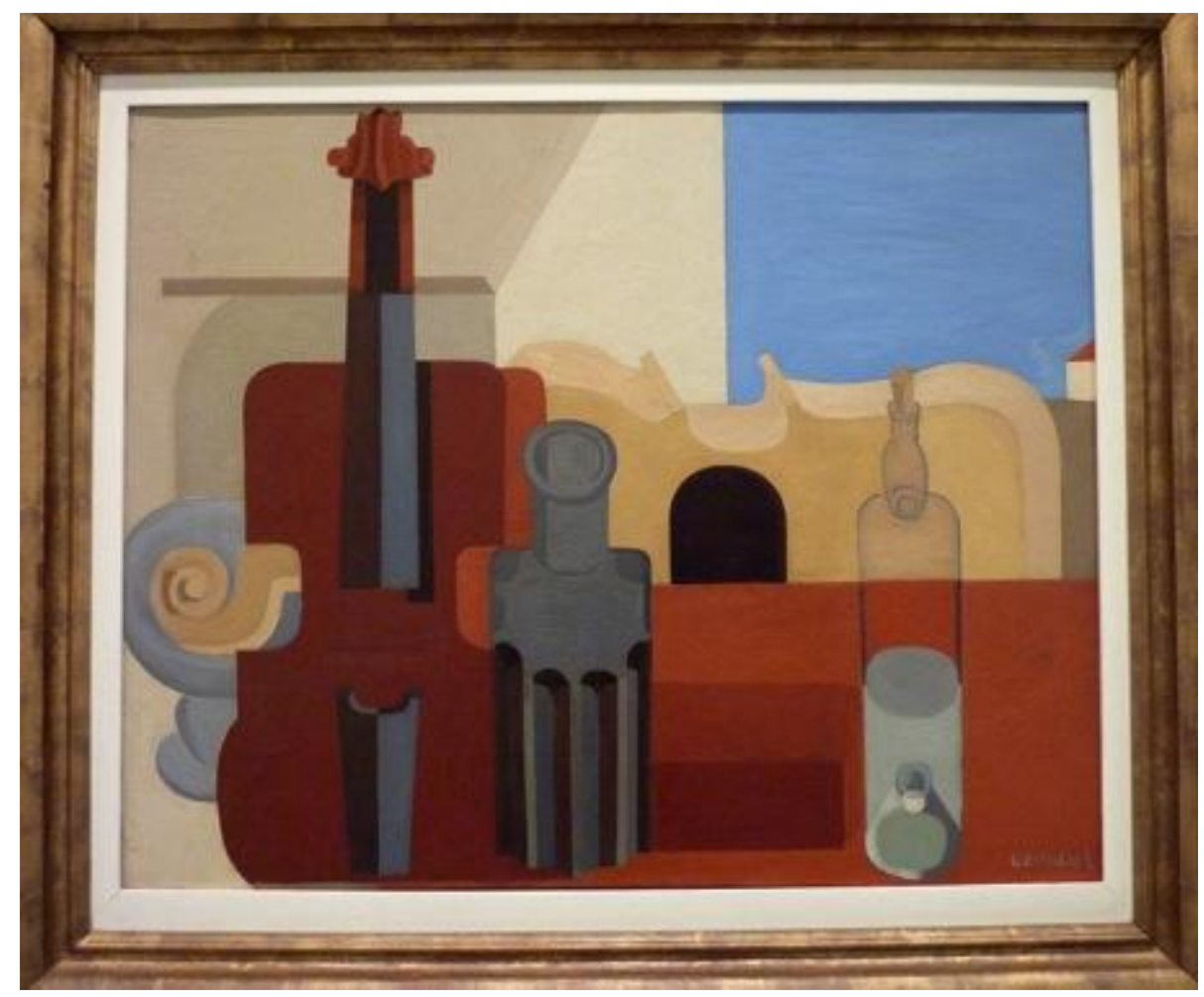

5. Amédée Ozenfant: Botella, frasco y violines 1920.

Una estructuración geométrica legible donde el observador-receptor, con un control visual sobre el conjunto, dota de significado a la obra, articulando las apariciones individuales mediante relaciones métricas y posicionales - un proceso puro con una lógica intrínseca intencionada, cuyas cualidades se envisten de una transparencia fenoménica (no literal)-. Unas interferencias entre el espacio profundo y el espacio superficial que siempre ocasionan múltiples lecturas - todavía desde la estabilidad y el orden- y que activan la experiencia de lo formal del sujeto receptor.

\section{Sistema relacional.}

En la construcción del espacio mediante la estratificación, no importa el objeto en sí -abstraído, sin perspectiva, cotidiano, puro, primario-, sino las relaciones o asociaciones entre elementos independientes que construyen un todo unitario. Unos estudios en los que, según afirma Le Corbusier: "la relación de los elementos [se produce] con vistas a crear en el cuadro un objeto único."

\footnotetext{
${ }^{5}$ Ozenfant, Amédée \& Jeanneret, Charles-Edouard: La peinture moderne. Paris: Les Éditions G. Crés \& Cie., 1925, p.168. (Traducción propia). Texto original: "La liaison des éléments en vue de créer dans le tableau un objet unique.". Véase también Ozenfant \& Jeanneret: Après le Cubisme, Paris: Éditions des Commentaires, 1918.
} 
Desde esta óptica, George Kepes analizará la posición-organización de los elementos en ese tipo de construcciones gráficas, señalando:

"El extremo inferior del plano gráfico ha representado el punto visual más próximo; en consecuencia, el grado de elevación de las unidades visuales indicaba posiciones espaciales de retroceso. "6

Y, al mismo tiempo, los variados objetos superpuestos se identifican con el plano que los contiene ayudando a establecer la relación espacial por su transparencia, según Kepes:

"Cuando contemplamos figuras superpuestas, reconocemos que la primera, o sea la superior, tiene dos significados espaciales, a saber, el de ella misma y el de detrás de ella misma. [...] Experimentamos las diferencias espaciales o profundidad.",

Se presenta un espacio estratificado de objetos independientes dentro de la unidad, y las relaciones entre ellos por medio de enlaces ópticos-mentales provocados por la transparencia y superposición de los planos paralelos en posiciones relativas que exploran una lógica de combinaciones posibles. Multiplicidad, simultaneidad y multifocalidad de recorridos visuales, desde una posición estática del observador-receptor, que pretende evocar una legibilidad mental, considerando que:

"El purismo parte del elemento formal primario y geométrico, porque sus reacciones sobre nuestro físico son relativamente simples, comparado al mundo de las asociaciones (...) es la sinfonía de las asociaciones provocadas por ellas quien mueve nuestra cenestesia y nuestro yo consciente. Hablando por lo tanto de elementos formales y coloridos y considerándolos como excitantes de una acción específica determinada, podemos crear el cuadro como una máquina. El cuadro es un dispositivo destinado a emocionarnos. Esta noción es fundamental en el Purismo." Ozenfant \& Jeanneret ${ }^{8}$

La unión entre la precisión (de la máquina) y la experiencia estética (excitación), entre la razón y la sensibilidad. Una emoción de orden intelectual provocada por la asociación de elementos que experimenta el sujeto receptor.

\footnotetext{
${ }^{6}$ Op. cit.: Kepes, G.: El lenguaje de la visión, p.111.

${ }^{7}$ Op. cit.: Kepes, G.: El lenguaje de la visión, p.112.

${ }^{8}$ Ozenfant \& Jeanneret: La peinture moderne. Paris: Les Éditions G. Crés \& Cie., 1925, pp.166-167. (Trad. propia). Texto original: "Le purisme part de l'élément formel primaire et géométrique, parce que ses réactions sur notre physique sont relativement simples, comparées au monde des associations (...) c'est la symphonie des associations provoquée per celles-ci qui émeut notre cénesthésie et notre moi conscient. Parlant donc des éléments formels et colorés et les considérant comme des excitants à action spécifique déterminée, on peut créer le tableau comme une machine. Le tableau est un dispositif destiné à nous émouvoir. Cette notion est fondamentale dans le purisme."
} 

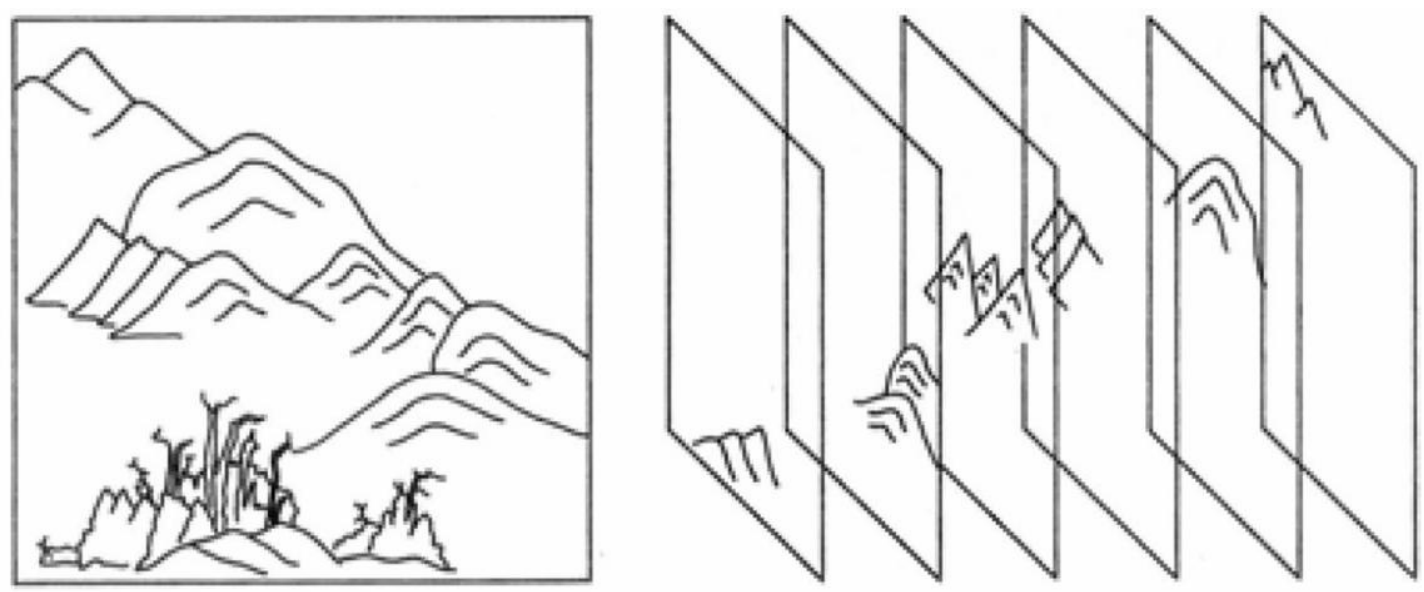

6. Diagramas de la superposición de montañas de la pintura de Li-Sheng, Dream Journey through the Xiao and Xiang Rivers, (ca.1170).

En comparación, el empleo de "planos paralelos" para la construcción del espacio (fig.6), iniciando el recorrido con pluralidad de puntos de vista, hasta obtener la sensación de que el espectador está ubicado en un lugar indeterminado, aéreo, desde el que contempla casi a vista de pájaro el panorama representado, conectando al observador con la elevación espiritual, lanzando la mirada hacia el paisaje lejano y extenso, es un fenómeno de la pintura china denominado shen yuan o "distancia profunda". Una técnica proveniente de los bajorrelieves chinos en los que se suceden y superponen diversas capas, con diferentes objetos opacos -sin transparencia literal- hasta crear el espacio. Tal y como se observa en el diagrama obtenido a partir de la pintura titulada Dream Journey through the Xiao and Xiang Rivers ${ }^{9}$.

Representaciones de la escena paisajística mediante la "acumulación" de "planos", siempre con una visión no geométrica ni limitada del espacio pictórico (tan característica de la perspectiva occidental). Todo ello en virtud de una mayor expresividad, más allá de la realidad, al ser reproducida una cualidad espiritual -vista por el ojo de la mente- y el espacio idealista habitado por otras realidades, por el eterno Qi o por el Tao.

Asimismo, este sistema de posicionamiento de los objetos, para dar sensación de profundidad sin límites (sin la utilización de la perspectiva), también puede observarse en los dibujos infantiles, donde, a falta de recursos conocidos para explicar lo que desea representar, el niño utiliza diversos aspectos visuales, tales como el fusionado del mundo tridimensional con el del plano gráfico bidimensional o el manejo de la combinación de planta y elevación. Aspectos similares a la multiplicidad de puntos de vista creados en los cuadros cubistas, que se alejan de la perspectiva única buscando la profundidad plana. Un recurso que también empleó Rem Koolhaas en la elevación-planta realizada para el concurso del Parque de la Villette, 1982 (una representación bidimensional de la elevación de cada franja de la planta del parque, con la que simula un gráfico tridimensional heterogéneo y simultáneo).

\footnotetext{
${ }^{9}$ Smith, Judith G., y Wen C. Fong (eds.): Issues of Authenticity in Chinese Painting. Nueva York: Department of Asian Art, The Metropolitan Museum of Art, 1999, p.273. La pintura original de Li, de la que se obtiene el presente diagrama, se encuentra en Tokyo National Museum.
} 


\section{Visión dinámica, experiencia narrativa espacio-temporal.}

El espacio se construye a través de la "estratificación", de la superposición de franjas verticales paralelas que avanzan y retroceden, englobándose y excluyéndose mutuamente en una relación de profundidad donde la mirada experimenta movimiento y tensiones desde una posición estática del observador.

La misma figura se ve como la más próxima o como la más alejada, es decir fluctúa, al concentrarse varias perspectivas espaciales divergentes en una sola captación visual; tal y como reconoce George Kepes:

"La transparencia representa una percepción simultánea de diferentes posiciones espaciales. El espacio no sólo retrocede sino que fluctúa en continua actividad. "10

Si bien, a diferencia de la posición de Rowe y Slutzky que mantienen al observador estático e alejado, Sigfried Giedion propone un espacio relacional activo. Tal y como apunta Detlef Mertins:

"Para ellos [Rowe y Slutzky], la transparencia se basó en un observador colocado en el eje de un plano bidimensional (un cuadro o la fachada de un edificio), inmóvil y desprovisto de pensamiento y acción. Sin embargo, para Giedion y sus colegas constructivistas, era la función de una espacialidad en cuatro dimensiones activada por un sujeto móvil y participativo. Giedion describió esto como un espacio "relacional" en lugar de un espacio de objetos. ${ }^{, 11}$

La visión múltiple -multifocal- supera la fijación estática de la perspectiva lineal -afocal o bifocal-. Una visión relativa -secuencial- y descentrada en una realidad estable, en la cual el espacio profundo se opone constantemente a la inferencia del espacio superficial, creando una tensión resultante que obliga a efectuar siempre nuevas interpretaciones de estructuras formales dentro de un todo, manteniendo la independencia de los objetos. Y a su vez, se van introduciendo actitudes dinámicas -no meramente contemplativas- que dan paso a la experimentación. Tal y como describe el propio Giedion al referirse a la transparencia literal de los planos del edificio de la Bauhaus:

"Hay una agrupación vertical y flotante de planos que satisface nuestra sensibilidad en favor de un espacio relacional, y hay una amplia transparencia [literal] que permite ver el interior y el exterior simultáneamente, de frente y de perfil, como en La arlesiana de Picasso, de 1911-1912: variedad de niveles de referencia, o de puntos de referencia, y simultaneidad; en resumen, la concesión del espacio-tiempo. ",2

Una percepción que será experimentada de modo narrativo en la arquitectura del movimiento moderno, como por ejemplo durante el recorrido de ascenso por la rampa ${ }^{13}$ (hasta el encuadre final del paisaje en la llegada a cubierta) de la villa Savoye, 1928, de Le Corbusier. Una secuencia sucesiva de planos o encuadres que se relacionan con el lenguaje cinematográfico y las técnicas de montaje, como capas enlazadas hasta obtener un significado global.

\footnotetext{
${ }^{10}$ Op. cit.: Kepes, G.: El lenguaje de la visión, p.114.

${ }^{11}$ Mertins, Detlef: Modernity Unbound: Other Histories of Architectural Modernity. Londres: Architectural Association AA Publications, 2011, pp.11 y 12. (Trad. propia) Texto original: "For them [Rowe y Slutzky], transparency relied on an observer stationed on axis with a two-dimensional plane (a painting or the façade of a building), immobile and devoid of thought and action. Yet for Giedion and his constructivist colleagues, it was a function of a four-dimensional spatiality activated by a mobile and participatory subject. Giedion described this as a "relational" space rather than a space of objects."

${ }^{12}$ Giedion, Sigfried: Espacio, tiempo y arquitectura : origen y desarrollo de una nueva tradición. Trad. y edición Jorge Sainz. Barcelona: Reverté, 2009, p.485. (Space, time and architecture : the growth of a new tradition. Cambridge: Harvard University Press, 1941).

${ }^{13}$ Una relectura de la promenade arquitectónica.
} 


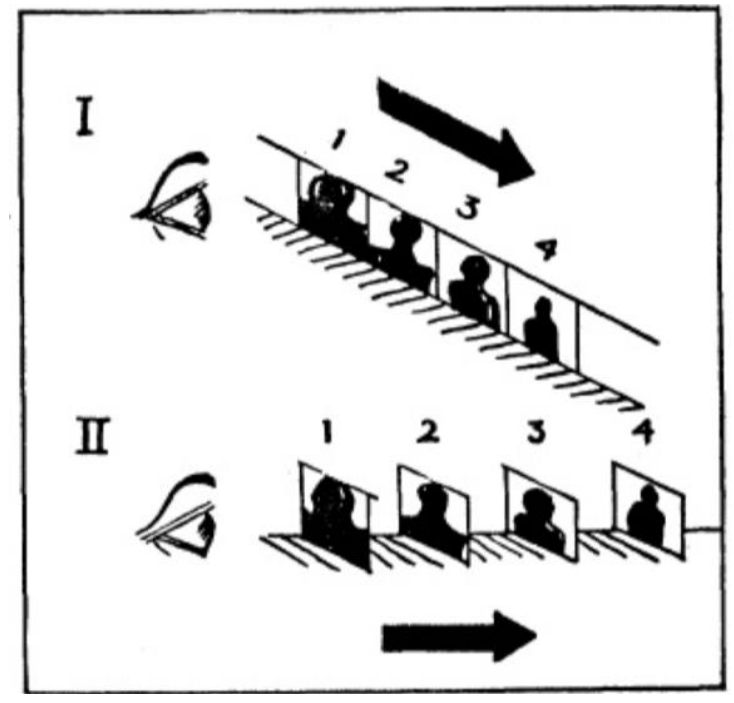

7. Eisenstein, Sergei: Esquema de percepción sobre la profundidad.

Tal y como esquematiza y argumenta Sergei Eisenstein (fig.7), en su libro El sentido del cine, para lograr la profundidad de una imagen:

"La vista puede disciplinarse, no para unir un cuadro a otro, como en nuestro fragmento [fig. I], sino para colocar uno sobre otro en capas. Esto produciría la sensación de ser arrojado dentro de una profundidad o la sensación de cuadros que se abalanzaran hacia el espectador. Imaginemos, por ejemplo, una serie de cuatro primeros planos de tamaño creciente, cada uno de una persona distinta y colocado en el centro de la toma. Una percepción natural de esta serie de cuadros no podría representarse en el diagrama superior sino en el inferior (fig. II)." 14

Una serie o secuencia de planos o capas superpuestas en continuidad, un establecimiento de discontinuidades físicas y enlaces psíquicos como en el montaje arquitectónico.

$\mathrm{Al}$ respecto, tal y como se sugiere, ante análisis actuales de la escena de la rampa:

"es posible que Le Corbusier tuviera la idea de tratar de insertar un montaje del tipo Eisenstein dentro de un corte clásico, si bien es extremadamente raro asociar estas dos gramáticas en una misma secuencia.” [...] "Por lo tanto podemos suponer que estas referencias a las teorías de montaje de Eisenstein -teorías inspiradas en la lección de Choisy- constituyen un enlace tangible al concepto del paseo arquitectónico con la Acrópolis. ",15

\footnotetext{
${ }^{14}$ Eisenstein, Sergei: El sentido del cine. México: ed. siglo XXI S.A., 1974, p.148. (The film sense. New York: Harcourt, Brace and Co., 1942).

${ }^{15}$ Fondation Le Corbusier: L'invention d'un architecte. Le voyage en Orient de Le Corbusier. Francia: ediciones de la Villette, 2013. (Trad. propia). Textos originales: "Il est aussi possible que Le Corbusier ait eu l'idée d'expérimenter l'insertion d'un montage de type Eisensteinien à l'intérieur du découpage classique, bien qu'il soit extrêmement rare d'associer ces deux grammaires dans una même séquence." p.411. "On peut donc supposer que ces références aux théories de montage d'Eisentein -théories inspirées par la leçon de Choisy- constituent un lien tangible du concept de la promenade architecturale avec l'Acropole." p.413.
} 

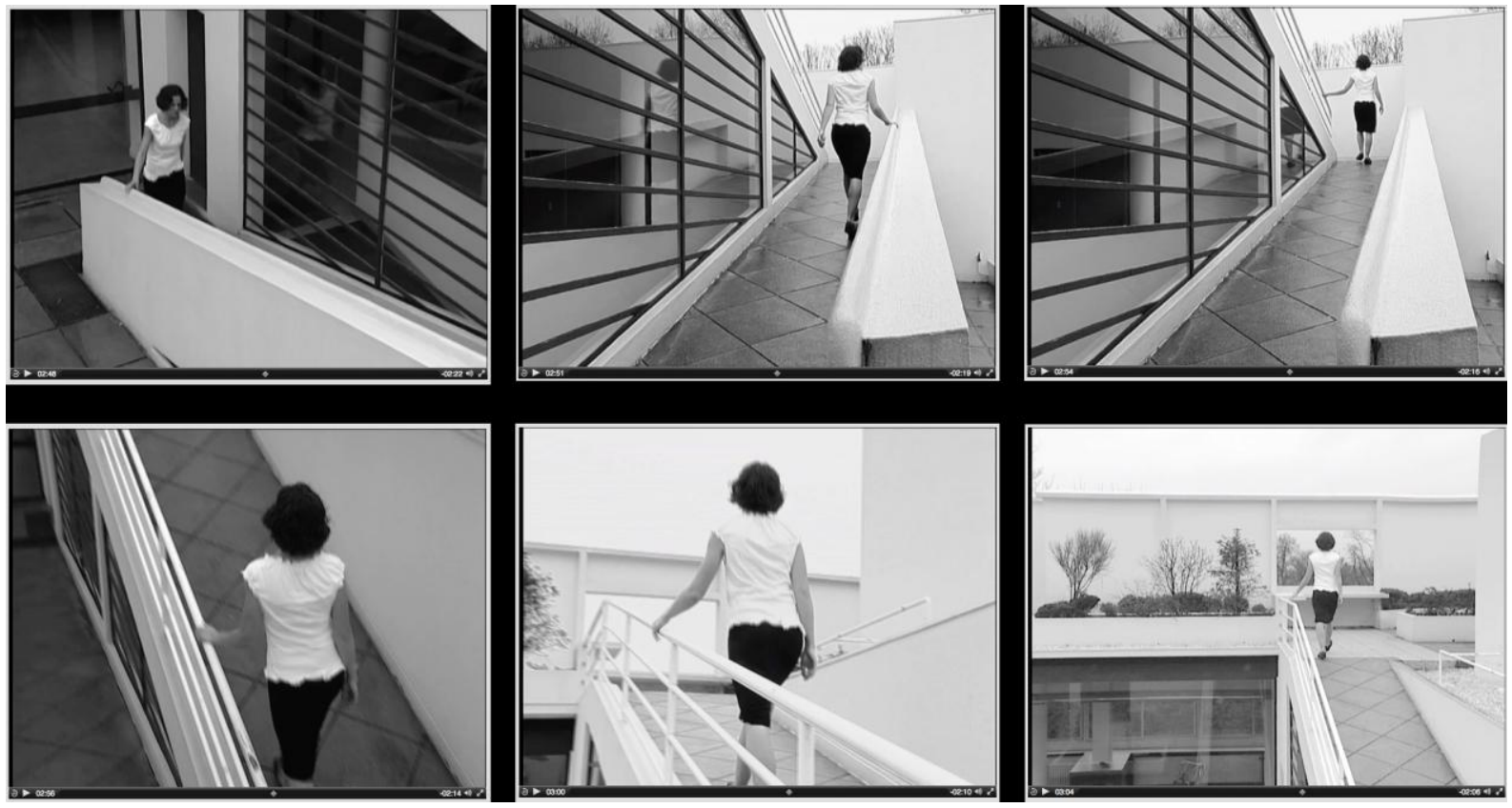

8. Selección de planos de la escena de la promenade arquitectónica del video de Matthew Roman titulado Savoye, 2004.

Un paseo que, en el nuevo montaje de la reinterpretación de la escena de la promenade arquitectónica de Matthew Roman ${ }^{16}$ (fig.8), transgrede el ángulo de 90 grados, definiendo la progresión ascendente de una mujer (subiendo por la rampa) utilizando una estructura diagonal, dando así un efecto cinematográfico que no es absolutamente bidimensional ni tridimensional.

En consecuencia, la construcción del espacio mediante la superposición de planos independientes, logra obtener una profundidad bidimensional y la multiplicidad de puntos de vista que, de modo similar a algunas vanguardias pictóricas del siglo XX, trata de introducir el tiempo en su definición plástica. Se genera, por tanto, una "fícción" de realidad volumétrica, y tal y como apuntaba Colin Rowe:

"El tiempo se convierte en el metrónomo del progreso y a sus aspectos seriales se les otorga una presencia acumulativa y dinámica, en tanto que, por otra, aunque secuencia y cronología se reconocen como los hechos que son, el tiempo, privado según esquemas experimentales. "17

El tiempo comienza a formar parte del espacio, es la cuarta dimensión. El tiempo antes absoluto, compacto, continuo, cuyo intervalo entre dos sucesos es homogéneo y separado del espacio -defendido por Aristóteles o Newton-, se transforma en un tiempo relativo, fragmentado, discontinuo -enunciado por Einstein en su "teoría de la relatividad"-. Y con ello, esta influencia de las teorías científicas de Einstein romperá con la composición estática, de centro único y punto de vista privilegiado clásico, dando paso a la posición relativa, donde cada "unidad" se concebiría como un centro independiente dentro de un espacio "relativista", de un espacio-tiempo referencial.

\footnotetext{
16 Véase video Savoye, 2004, de Matthew Roman, en el archivo de video-estudio digital de Cinematic Mapping of Cambridge. http://expressivespace.org/CMC/DIGIS-VE-22.html

${ }^{17}$ Rowe, Colin y Koetter, Fred: Ciudad Collage, Barcelona: Gustavo Gili, 1981, p.140. Texto original: "On the one hand time becomes the metronome of progress, its serial aspects are given cumulative and dynamic presence; while, on the other, though sequence and chronology are recognized for the facts which they are, time, deprived of some of its linear imperatives." Rowe, Colin y Koetter, Fred: Collage City. Cambridge, Massachusetts: MIT Press, 1978, pp.143-144.
} 
Le Corbusier empleó la superposición de estratos, de elementos "autónomos" y "espaciados", fragmentos de experiencias discontinuas en posiciones relativas, manteniendo la unidad compositiva, y ampliando los puntos de vista, tanto en el plano vertical (como se ha visto anteriormente) como horizontal, siendo el modelo configurado mediante el empleo de un sistema de plantas libres, o forjados de hormigón armado, extendidas verticalmente mediante superposición, denominado Estructura Dom-ino -palabra formada por domus (casa) e innovation (innovación)-, de 1914, uno de los primeros acercamientos de referencia de Le Corbusier a la producción de una "teoría sistemática de composición arquitectónica de posición relativa" vinculada al concepto de "superposición" como "estratificación".

La cohesión y continuidad clásicas - cuyas partes remiten al todo perdiendo autonomía o independencia-, pasan a la fragmentación y discontinuidad modernas -cuyos elementos desde su posición relativa hacen presente la autonomía de las partes articuladas que constituyen la unidad-.

Cada planta libre, o plano horizontal independiente, será un elemento sujeto a la fuerza de vectores verticales que mantendrá una organización fija dentro del conjunto, posibilitando la compresión de la arquitectura en términos tradicionales como orden y geometría elemental -racional-, y al mismo tiempo, una definición espacial "abstracta" y "depurada", propia de la arquitectura del movimiento moderno, si bien en el caso de Estructura Dom-ino, la carencia de enlaces entre los planos horizontales provocará la rigidez de su sección, y tal como citará Federico Soriano "A la planta libre le falta profundidad"18. Una cuestión que Le Corbusier resolverá mediante la ruptura de los suelos abstractos y neutrales -con la inclusión de nuevos elementos o mecanismos, como la doble altura o la rampa, que enlazaba los espacios con un concepto lineal y narrativo del tiempo- y, posteriormente, --con figuras orgánicas que cualificaban el espacio moderno con tensiones específicas-.

\footnotetext{
${ }^{18}$ Soriano, Federico: "Planta fluctuante". Fisuras de la cultura contemporánea, n.3 1/3, 1995, p.67. Véase también en "Hacia una definición de la planta profunda, de la planta anamórfica, y la planta fluctuante.”, El Croquis, n.81-82, 1996, p.7.
} 


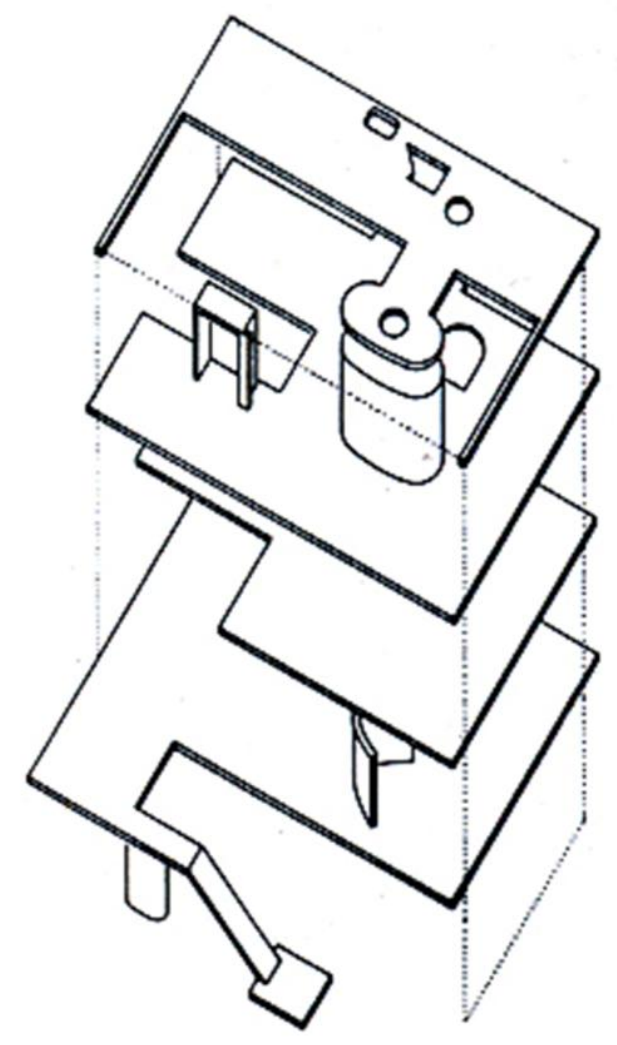

9. Colin Rowe y Robert Slutzky: Descomposición horizontal, en una serie de planos paralelos, de la Villa Garches, 1926, de Le Corbusier.

Así, los planos horizontales -suelos- serán operados hasta conseguir aumentar la dimensión espacial, de la 3D a la 4D, introduciendo el espacio-tiempo. Una planimetría referencial de experiencias fragmentadas - no compacta sino discontinua-, posiciones vinculantes con una voluntad estabilizadora y de control, una superposición visual de distintos planos que desintegran virtualmente la caja. Evocar "l'espace indicible" provocado por el espíritu y que no se puede describir con palabras, donde la plástica expresa la plenitud de un volumen según las exigencias del espíritu. Tal y como expresaba Le Corbusier:

"Entonces surge una profundidad sin límites, que borra los muros, ahuyenta las presencias contingentes: realiza el milagro del espacio indecible. "20

Una nueva "espacialidad plana" donde la arquitectura se expande hacia nuevas dimensiones, hacia un espacio continuo. Así, el movimiento de la mirada, y la sucesión o secuencia de espacios, es conducido también por un recorrido arquitectónico narrativo, bien sea físicamente por ejemplo al desplazarnos por la rampa, o virtualmente mediante la visión dinámica generada por la superposición y transparencia de los planos separados temporalmente y sus relaciones geométricas inestables.

${ }^{19}$ Le Corbusier: "L'espace indicible" escrito en 1945, publicado en L'Architecture d'Aujourd'hui, n. especial "Art", 1946, pp.9-17. ("El espacio indecible”. Trad. Fernando Álvarez, corrección Carlota Socías, DC Revista de crítica arquitectónica, n.1, 1998).

${ }^{20}$ Ibíd.: Le Corbusier: "El espacio indecible”, p.47; Texto original: "Alors une profondeur sans bornes s'ouvre, efface les murs, chasse les présences contingentes, accomplit le miracle de l'espace indicible." 


\section{Hacia el impacto emocional.}

Estos mecanismos formales, expuestos precedentemente, -que en sus orígenes fueron controlados, claros en la exposición de sus relaciones y de lectura correcta- (experimentados por Le Corbusier y posteriormente redirigidos por Rowe y Slutzky en atracción y defensa de las propiedades formales de obras arquitectónicas y pictóricas), acabaran transformándose en recursos manipulables, deformables, e ilegibles (utilizados por Peter Eisenman). En este sentido, tal y como cita Jeffrey Kipnis en su artículo $P$-Tr's Progress:

"A efectos prácticos, todas las intervenciones formales de Eisenman derivan del tratamiento que Rowe/Slutzky hacen de la Transparencia Fenomenológica." 21

Ambos buscarán la experimentación de lo formal como acto que provoque una emoción de orden intelectualafectivo en el sujeto receptor - estático o dinámico-, si bien, en los tiempos actuales, se añadirá la complejidad en su lectura -rozando o alcanzando lo ilegible-, turbándose las asociaciones entre elementos, pero manteniendo mecanismos como la superposición, estratificación, articulación, etc. En cierto modo, la transparencia se convierte en traslucidez o borrosidad, y la lectura en afecto. Tal y como explica Peter Eisenman:

"Afectar tiene que ver con el modo en que formas particulares de efectos arquitectónicos, tropos, retórica, pueden desplazar nuestra experiencia convencional o esperada del espacio., 22

De lo que se deriva que, la liberación de la legibilidad -hacia lo topológico- conduce a procesos sin un sentido direccional preciso, sin transmisión directa de las relaciones, y repletos de secuencias ambivalentes. Extraños seccionados y combinaciones delirantes que promueven las experiencias inusuales (sensaciones imprevistas, no entendibles o no intencionadas) frente a experiencias inteligibles (lecturas formales, claras, leíbles). Efectos formales cada vez más complejos y avanzados, y no por ello menos interesantes o propositivos que sus antecesores.

\section{Procedencia de imágenes}

1, 3 y 9. Rowe, Colin y Slutzky, Robert en Transparency, 1955-1956; 2. Fondation Le Corbusier (C) FLC/ADAGP; 4 y 5. Fotografías propias. Kunstmuseum Basel; 6. Autor desconocido en Issues of Authenticity in Chinese Painting; 7. Sergei Eisenstein en The film sense, 1942; 8. Montaje propio sacado del video de Matthew Roman publicado en http://expressivespace.org/CMC/DIGIS-VE-22.html

\section{Bibliografía/referencias}

Benjamin, Andrew (et al.): Blurred zones: investigations of the interstitial. Eisenman Architects, 1988-1998. Nueva York: Monacelli Press, 2002.

Eisenstein, Sergei: The film sense. New York: Harcourt, Brace and Co., 1942.

Fondation Le Corbusier: L'invention d'un architecte. Le voyage en Orient de Le Corbusier. Francia: ediciones de la Villette, 2013.

\footnotetext{
${ }^{21}$ Kipnis, Jeffrey: “P-Tr's Progress". En El Croquis, n.83, "Peter Eisenman 1990-1997”. Madrid: ed. El Croquis, 1997, p.38.

${ }^{22}$ Eisenman, Peter en Benjamin, Andrew (et al.): Blurred zones: investigations of the interstitial. Eisenman Architects, 19881998. Nueva York: Monacelli Press, 2002, p. 6. Texto original: "Affect is concerned with the way particular forms of architectural effects, tropes, rethoric, can displace our conventional or expected experience of space.".
} 
Giedion, Sigfried: Space, time and architecture : the growth of a new tradition. Cambridge: Harvard University Press, 1941.

Kepes, Gyorgy: Language of vision. Chicago: Paul Theobald, 1944.

Kipnis, Jeffrey: “P-Tr's Progress”. En El Croquis, n.83, “Peter Eisenman 1990-1997”. Madrid: ed. El Croquis, 1997.

Le Corbusier: "L’espace indicible” 1945. En L'Architecture d'Aujourd'hui, n. especial “Art”, 1946.

Mertins, Detlef: Modernity Unbound: Other Histories of Architectural Modernity. Londres: Architectural Association AA Publications, 2011.

Ozenfant \& Jeanneret: La peinture moderne. Paris: Les Éditions G. Crés \& Cie., 1925.

Ozenfant \& Jeanneret: Après le Cubisme, Paris: Éditions des Commentaires, 1918.

Rowe, Colin y Slutzky, Robert: “Transparency: Literal and Phenomenal”, 1955-1956. En Perspecta n.8, Yale Architectural Journal, 1963.

Rowe, Colin y Koetter, Fred: Collage City. Cambridge, Massachusetts: MIT Press, 1978.

Smith, Judith G., y Wen C. Fong (eds.): Issues of Authenticity in Chinese Painting. Nueva York: Department of Asian Art, The Metropolitan Museum of Art, 1999.

Soriano, Federico: "Planta fluctuante". En Fisuras de la cultura contemporánea, n.3 1/3, 1995. 